\title{
Association between metabolic syndrome and clinicopathological features of papillary thyroid cancer
}

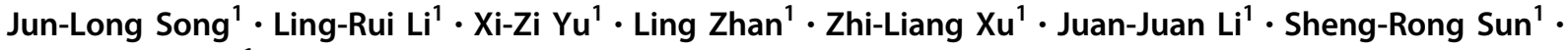 \\ Chuang Chen (1) ${ }^{1}$
}

Received: 6 April 2021 / Accepted: 6 November 2021 / Published online: 26 November 2021

(c) The Author(s), under exclusive licence to Springer Science+Business Media, LLC, part of Springer Nature 2021

\begin{abstract}
Background Metabolic syndrome (MetS) was a risk factor for papillary thyroid cancer (PTC). Whether MetS impacts the aggressiveness of PTC is still unclear. We carried out this study to clarify this issue.

Methods We evaluated 745 consecutive PTC patients treated with surgery. Patients were divided into three groups based on their number of MetS components: patients without any MetS components, patients with 1-2 MetS components, and patients with 3-5 MetS components. The clinical features and histological aggressiveness of PTC at the time of diagnosis were evaluated.

Results A total of 745 patients were included in this study. And, 145 patients had three or more metabolic components and were diagnosed as MetS. MetS was a risk factor for larger tumors ( $\mathrm{OR}=2.29,95 \% \mathrm{CI}$ : 1.31-4.03), more lymph node metastasis $(\mathrm{OR}=1.97,95 \% \mathrm{CI}: 1.11-3.51)$, and later clinical stage $(\mathrm{OR}=7.92,95 \% \mathrm{CI}: 1.59-39.34)$ after correction for age, sex, and thyroid-stimulating hormone (TSH) level and body mass index (BMI).

Conclusion In our hospital-based cohort study MetS was associated with the aggressiveness of PTC. This association was still significant after adjusting for age, sex, TSH, and BMI.
\end{abstract}

Keywords Thyroid cancer $\cdot$ Metabolic syndrome $\cdot$ Clinicopathological features $\cdot$ Aggressiveness

\section{Instruction}

The incidence of thyroid cancer in China has increased significantly over the past two decades [1]. It is more common in women, with a 3:1 female-to-male ratio. Papillary thyroid cancer (PTC) is the most common histologic subtype of thyroid cancer, accounting for $85 \%$ of new cases, and has the best prognosis. Increased detection of subclinical thyroid cancers by increased utilization of ultrasonography and fineneedle biopsies may partly account for the rise in the incidence of thyroid cancer [2,3]. However, this does not sufficiently explain the increase in the incidence of large tumors, in all size categories. Therefore, the effect of other

These authors contributed equally: Jun-Long Song, Ling-Rui Li

Chuang Chen

chenc0678@126.com

1 Department of Breast and Thyroid Surgery, Renmin Hospital of Wuhan University, Wuhan, Hubei 430060, PR China modifiable factors including changes in lifestyle and environmental factors cannot be ignored.

Metabolic syndrome (MetS), which indicates a cluster of metabolic abnormalities including central obesity, hyperglycemia, hypertension, and dyslipidemia, has been considered to be a risk factor of a variety of cancers, including thyroid cancer [4]. The major mechanism of MetS is insulin resistance. Several studies have investigated the association between MetS and thyroid cancer [4-6]. Park et al. followed $9,890,917$ adults for 7 years and found that the thyroid cancer risk was higher in the MetS group than in the nonMetS group $(\mathrm{HR}=1.15 ; 95 \%$ CI 1.13-1.17) [5]. A metaanalysis by Yin et al. also showed metabolic components, including hyperglycemia, high BMI, and hypertension significantly increased the thyroid cancer risk [4].

The prevalence of obesity and unhealthy metabolic status such as MetS has markedly increased in recent years. Substantial portion of PTC patients suffer from MetS. MetS is a reversible condition associated with the modern western lifestyle. It is important to clarify whether MetS affect the clinical features and prognosis of PTC. However, to the best of our knowledge, few studies 
focused on this issue. Therefore, we carried out this study to investigate the association between MetS and the clinicopathologic features of PTC.

\section{Materials and methods}

\section{Population}

A hospital-based cohort of patients that were diagnosed with PTC at the Renmin Hospital of Wuhan University from April, 2020 to February, 2021 were included in this study. All of the included patients received surgery treatment. Patients were excluded if there was no documentation of their past history of disease and/or if they were diagnosed with medullary or anaplastic thyroid cancer or follicular thyroid cancer. This study was performed with the approval of the ethics committee of the Renmin Hospital of Wuhan University (NO. WDRY2020-K231), and all of the participating patients were provided written informed consent for their clinical data to be reviewed and applied in this study.

\section{Data collection}

A comprehensive questionnaire was used to obtain the basic information, including medical history, family history of chronic diseases, current medication use, and smoking and drinking status. Height, weight, waist circumference, and blood pressure were measured based on standardized protocols. Height and body weight were measured without shoes and heavy clothes. Waist circumference, midway between the base of the costal arch and the top of the iliac crest, was measured in upright standing subjects. Blood pressure was taken using the same electronic monitor in the center in a seated position after at least $5 \mathrm{~min}$ of rest. Blood sampling was conducted after overnight fasting. Biochemical measurements, including thyroid-stimulating hormone (TSH), fasting blood glucose (FBG), triglycerides (TG), total cholesterol, high-density lipoprotein cholesterol (HDL-C), and thyroglobulin 1 month after surgery were measured utilizing the same laboratory techniques of the center.

\section{Diagnosis and definition}

All of the patients received surgical treatment for the primary tumor. Thyroid surgery was performed by experienced surgeons who had 5-20 years of experience. The central lymph node dissection was routinely performed on these patients. Intraoperative frozen section was performed on all patients to determine nodule malignancy. The inclusion of lateral lymph node dissection was performed based on preoperative imaging results, fine-needle aspiration, and intraoperative exploration. The central neck refers to levels VI and VII; whereas the lateral neck includes levels II, III, IV, and V in the present study.

A routine pathologic diagnosis was performed on the specimens of the thyroid and fatlike tissues with $2-3 \mu \mathrm{m}$ sections. Hematoxylin and eosin (HE) staining combined with or without immunohistochemistry was performed to determine the pathologic types. Solitary focus means only one tumor in the thyroid, and multiple foci mean two or more foci limited to the thyroid. Thyroid cancer was staged according to 8th American Joint Committee on Cancer (AJCC) editions.

The criteria for the diagnosis of MetS in our study was provided by the Chinese Diabetes Society. MetS was defined by the presence of 3 to 5 of the following variables: (1) abdominal adiposity (defined as waist circumference $\geq 90 \mathrm{~cm}$ in Chinese men and waist circumference $\geq 85 \mathrm{~cm}$ in Chinese women), (2) TG concentration $\geq 1.70 \mathrm{mmol} / \mathrm{L}$, (3) $\mathrm{HDL}$ concentrations $<1.04 \mathrm{mmol} / \mathrm{L}$, (4) blood pressure $\geq 130 /$ $85 \mathrm{mmHg}$ or previous hypertension diagnosis, which were described as raised blood pressure, and (5) fasting glycemia $>6.1 \mathrm{mmol} / \mathrm{L}$ or previous type 2 diabetes diagnosis.

\section{Statistical analysis}

All of the statistical analyses were performed using Stata version 15.1 (Stata Corp, College Station, Texas). Continuous variables were described as the mean $(x \pm \mathrm{SD})$. Categorical variables were described by using percentages. The distribution of clinical and pathologic features of the patients with or without MetS were evaluated and compared using chi-square tests for categorical variables and the student's $t$-test (age) or rank-sum test for continuous variables (tumor size, TSH, BMI, and thyroglobulin). Test for interaction was used to analyze the interaction effect of gender and age and MetS on the clinicopathological characteristics of PTC. Univariate and multivariate binary logistic analyses were used to explore the associations between MetS and clinicopathological characteristics of PTC. In the multivariate logistic regress model, age, sex, TSH, and BMI were adjusted. Furthermore, we also analyzed the association between different numbers of MetS components and the clinicopathological characteristics of PTC. All statistical tests were two-tailed, and a $p$-value $<0.05$ was considered statistically significant.

\section{Results}

\section{Characteristics of participants}

A total of 745 patients, including 181 males and 564 females with PTC, participated in this study. Table 1 describes the pathological characteristics of PTC and the 
Table 1 Clinicopathological characteristics of included patients

\begin{tabular}{|c|c|c|}
\hline Characteristics & Patients & $\%$ \\
\hline \multicolumn{3}{|l|}{ Sex } \\
\hline Male & 181 & 24.30 \\
\hline Female & 564 & 75.70 \\
\hline Age $($ mean \pm SD $)$ & $43.98 \pm 11.71$ & I \\
\hline BMI & $23.50 \pm 3.24$ & I \\
\hline \multicolumn{3}{|l|}{ Subtype } \\
\hline Classic variant & 539 & 72.35 \\
\hline Follicular variant & 164 & 22.01 \\
\hline Other variant & 42 & 5.64 \\
\hline Tumor size $($ mean $\pm \mathrm{SD})$ & $1.02 \pm 0.75 \mathrm{~cm}$ & I \\
\hline$\leq 1 \mathrm{~cm}$ & 473 & 63.49 \\
\hline$>1 \mathrm{~cm}$ & 272 & 36.51 \\
\hline Number of removed lymph node median (Q1-Q3) & $7(3-11)$ & l \\
\hline \multicolumn{3}{|l|}{ Lymph node metastasis } \\
\hline N0 & 397 & 53.29 \\
\hline N1 & 348 & 46.71 \\
\hline \multicolumn{3}{|l|}{ AJCC Stage } \\
\hline I & 702 & 94.23 \\
\hline II/III & 43 & 5.77 \\
\hline \multicolumn{3}{|l|}{ Multifocal tumor } \\
\hline No & 541 & 72.62 \\
\hline Yes & 204 & 27.38 \\
\hline \multicolumn{3}{|l|}{ Blood pressure $\geq 130 / 85 \mathrm{mmHg}$} \\
\hline No & 426 & 57.18 \\
\hline Yes & 319 & 42.82 \\
\hline \multicolumn{3}{|l|}{ Diabetes } \\
\hline No & 687 & 92.21 \\
\hline Yes & 58 & 7.79 \\
\hline \multicolumn{3}{|l|}{ High TG } \\
\hline No & 567 & 76.11 \\
\hline Yes & 178 & 23.89 \\
\hline \multicolumn{3}{|l|}{ Low HDL-C } \\
\hline No & 550 & 73.83 \\
\hline Yes & 195 & 26.17 \\
\hline \multicolumn{3}{|l|}{ Abdominal adiposity } \\
\hline No & 567 & 76.11 \\
\hline Yes & 178 & 23.89 \\
\hline \multicolumn{3}{|l|}{ Number of MetS components } \\
\hline 0 & 272 & 36.51 \\
\hline $1-2$ & 328 & 44.03 \\
\hline $3-5$ & 145 & 19.46 \\
\hline
\end{tabular}

Values are expressed as mean \pm standard deviation or number (\%)

$S D$ Standard deviation, BMI Body mass index, AJCC American Joint Committee on Cancer, $T G$ Triglycerides, $H D L-C$ High-density lipoprotein cholesterol, and MetS Metabolic syndrome

metabolic characteristics of the participants at recruitment. Most of the patients were diagnosed with classic variant $(72.35 \%)$ or follicular variant of PTC $(22.01 \%)$. Other special variants such as the tall cell variant were rare $(5.64 \%)$. The mean age of the participants was 43.98 (14-85). The mean dimension of the primary tumor was $1.02 \mathrm{~cm}(0.08-6 \mathrm{~cm})$, and in most cases, was less than $4 \mathrm{~cm}$. About $63.49 \%$ of the participants had a tumor less than $1 \mathrm{~cm}$. All of the included participants received central lymph node dissection according to our surgical protocol. The median number of dissected lymph node was 7 (Q1-Q3: 3-11). And lateral lymph node dissection was performed in 63 participants $(8.46 \%)$. A total of 348 $(46.71 \%)$ participants had metastatic lymph nodes. About $27.38 \%$ of the tumors were multifocal. A total of 43 patients had stage II/III disease while 702 patients had stage I disease. However, only about 50\% (375/745) of the patients came back to our center for postsurgical review due to the pandemic of COVID-19. A total of 116 patients received radioactive iodine therapy. Almost $43 \%$ of the patients had blood pressure $\geq 130 / 85 \mathrm{mmHg}$. And $7.79 \%$ of the patients had diabetes. High TG was detected in $23.89 \%$ (178/745) of the participants. While low HDL was detected in $26.17 \%$ (195/745) of the participants. A total of 178 of the participants had abdominal adiposity. A total of 328 (44.03\%) patients had one or two metabolic components. And $145(19.46 \%)$ patients had three or more than three metabolic components and were diagnosed as MetS.

\section{Relationship between MetS and clinicopathological features}

Clinical data and tumor characteristics, according to the presence or absence of MetS, are reported in Table 2. More men suffered from MetS than women. There was no significant difference in TSH level between those patients with and without MetS. The patients with MetS were older $(p=0.000)$, and had larger tumor $(p=0.001)$, more lymph node metastasis $(p=0.048)$ and later AJCC stage $(p=0.000)$ than the patients without any components of MetS. More patients were diagnosed with other rare variants of PTC in MetS group $(p$ $=0.000$ ). The patients with MetS had similar multifocal diseases with patients without MetS. The proportion of patients received radioactive iodine therapy was similar between different groups. There were no significant differences in thyroglobulin level 1 month after surgery between different groups $(p=0.918)$. The cases with one or two metabolic components showed intermediate figures.

Univariate and multivariate analyses were performed to detect the correlation between MetS and clinicopathological features. As shown in Table 3, in univariate analysis, MetS was associated with larger tumor $(\mathrm{OR}=2.18,95 \%$ CI: 1.44-2.03), more lymph node metastasis $(\mathrm{OR}=1.60,95 \% \mathrm{CI}: 1.07-2.41)$, and later stage $(\mathrm{OR}=7.16,95 \%$ CI: 2.31-22.18). Furthermore, after correction for age, sex, TSH, and BMI, MetS was still a risk factor for larger tumors $(\mathrm{OR}=2.29,95 \% \mathrm{CI}$ : 1.31-4.03), more lymph node metastasis $(\mathrm{OR}=1.97$, 95\% CI: $1.11-3.51)$, and later stage $(\mathrm{OR}=7.92,95 \% \mathrm{CI}$ : 1.59-39.34). Those patients with one or two metabolic components had higher risks for larger tumor $(\mathrm{OR}=1.18$, 95\% CI:1.00-2.19). However, there were no differences 
Table 2 Clinicopathologic characteristics for patients with and without metabolic syndrome

\begin{tabular}{|c|c|c|c|c|}
\hline \multirow[t]{2}{*}{ Characteristics } & \multicolumn{2}{|l|}{ Non-MetS } & \multirow{2}{*}{$\begin{array}{l}\text { MetS } \\
\geq 3 \text { components }\end{array}$} & \multirow[t]{2}{*}{$p$} \\
\hline & 0 components & $1-2$ components & & \\
\hline \multicolumn{5}{|l|}{ Sex } \\
\hline Male & $24(8.82)$ & $81(24.70)$ & $76(52.41)$ & 0.000 \\
\hline Female & $248(91.18)$ & $247(75.30)$ & $69(47.59)$ & \\
\hline Age $($ mean \pm SD $)$ & $40.40 \pm 11.62$ & $45.86 \pm 11.70$ & $46.63 \pm 10.29$ & 0.000 \\
\hline$<55$ & $242(88.97)$ & $244(74.39)$ & $108(74.48)$ & 0.000 \\
\hline$\geq 55$ & $30(11.03)$ & $84(25.61)$ & $37(25.52)$ & \\
\hline TSH $($ mean $\pm \mathrm{SD}, \mu \mathrm{IU} / \mathrm{mL})$ & $2.68 \pm 7.14$ & $2.11 \pm 3.10$ & $2.66 \pm 4.68$ & 0.035 \\
\hline $\begin{array}{l}\text { Thyroglobulin (mean } \pm \text { SD, } \\
\text { ng/mL) }\end{array}$ & $6.77 \pm 39.39$ & $4.98 \pm 16.85$ & $3.04 \pm 4.29$ & 0.918 \\
\hline BMI $($ mean \pm SD) & $21.71 \pm 2.14$ & $23.49 \pm 2.89$ & $26.85 \pm 3.06$ & 0.000 \\
\hline \multicolumn{5}{|l|}{ Pathological subtype } \\
\hline Classic variant & $221(81.25)$ & $212(64.63)$ & $106(73.10)$ & 0.000 \\
\hline Follicular variant & $38(13.97)$ & $100(30.49)$ & $26(17.93)$ & \\
\hline Other & $13(4.78)$ & $16(4.88)$ & $13(8.97)$ & \\
\hline \multicolumn{5}{|l|}{ Tumor size(cm) } \\
\hline Mean \pm SD & $0.95 \pm 0.73$ & $1.03 \pm 0.77$ & $1.14 \pm 0.73$ & 0.001 \\
\hline$\leq 1$ & $192(70.59)$ & $205(62.50)$ & $76(52.41)$ & 0.001 \\
\hline$>1$ & $80(29.41)$ & $99(37.50)$ & $69(47.59)$ & \\
\hline \multicolumn{5}{|l|}{ Lymph node metastasis } \\
\hline No & $152(55.88)$ & $181(55.18)$ & $64(44.14)$ & 0.048 \\
\hline N1 & $120(44.12)$ & $147(44.82)$ & $81(55.86)$ & \\
\hline \multicolumn{5}{|l|}{ AJCC Stage } \\
\hline I & $268(98.53)$ & $303(92.38)$ & $131(90.34)$ & 0.000 \\
\hline II/III & $4(1.47)$ & $25(7.62)$ & $14(9.66)$ & \\
\hline \multicolumn{5}{|l|}{ Multifocal tumor } \\
\hline No & $198(72.79)$ & $247(75.30$ & $96(66.21)$ & 0.123 \\
\hline Yes & $74(27.21)$ & $81(24.70)$ & 49 (33.79) & \\
\hline \multicolumn{5}{|l|}{ Radioactive iodine therapy } \\
\hline No & $231(84.93)$ & $281(85.67)$ & $117(80.69)$ & 0.372 \\
\hline Yes & $41(15.07)$ & $47(14.33)$ & $28(19.31)$ & \\
\hline
\end{tabular}

Values are expressed as mean \pm standard deviation or number $(\%)$

SD Standard deviation, TSH Thyroid-stimulating hormone, BMI Body mass index, AJCC American Joint Committee on Cancer, and MetS Metabolic syndrome in lymph node metastasis, multifocal tumor, and AJCC stage between patients with one or two metabolic components and patients without any metabolic components.

\section{Relationship between metabolic components and clinicopathological features}

We further analyzed the relationship between each of the five metabolic components and the clinicopathological features. As shown in Fig. 1, after adjusted for age, sex, TSH and BMI, blood pressure $\geq 130 / 85 \mathrm{mmHg}$ was associated with larger tumor $(\mathrm{OR}=1.65,95 \% \mathrm{CI}$ : $1.17-2.32)$, lymph node metastasis $(\mathrm{OR}=1.41,95 \% \mathrm{CI}: 1.00-2.02)$ and later AJCC stage $(\mathrm{OR}=3.99,95 \% \mathrm{CI}$ : $1.56-10.21)$.
Diabetes, TG and abdominal adiposity had no significant association with any of the clinicopathological features. Low HDL was associated with larger tumor $(\mathrm{OR}=1.55$, 95\% CI: 1.05-2.28) and lymph node metastasis (OR = $1.92,95 \%$ CI: $1.29-2.86)$.

\section{Discussion}

To our knowledge, this is the first study to determine the association between MetS and clinicopathological features of PTC. This hospital-based cohort study revealed that patients with MetS tended to have larger tumors. Such risk is likely to be rather the expression of a general 
Table 3 Univariate and multivariate ORs of metabolic syndrome for prognostic factors of papillary thyroid cancer
Fig. 1 Multivariate analysis for relationship between five metabolic components and clinicopathological features

\begin{tabular}{llllll}
\hline & $\begin{array}{l}\text { Non-MetS } \\
\text { 0 components }\end{array}$ & $\begin{array}{l}\text { Non-MetS } \\
(1-2 \text { components })\end{array}$ & $P$ & $\begin{array}{l}\text { MetS } \\
(\geq 3 \text { components })\end{array}$ & $P$ \\
\hline Univariate ORs & & & & & \\
Tumor size & 1 & $1.44(1.02-2.03)$ & 0.037 & $2.18(1.44-2.03)$ & 0.000 \\
Lymph node metastasis & 1 & $1.03(0.74-1.42)$ & 0.864 & $1.60(1.07-2.41)$ & 0.023 \\
Multifocal tumor & 1 & $0.88(0.61-1.27)$ & 0.484 & $1.37(0.88-2.11)$ & 0.161 \\
AJCC stage & 1 & $5.53(1.90-16.09)$ & 0.002 & $7.16(2.31-22.18)$ & 0.000 \\
Multivariate ORs* & & & & & \\
Tumor size & 1 & $1.18(1.00-2.19)$ & 0.049 & $2.29(1.31-4.03)$ & 0.004 \\
Lymph node metastasis & 1 & $1.29(0.87-1.90)$ & 0.205 & $1.97(1.11-3.51)$ & 0.020 \\
Multifocal tumor & 1 & $0.85(0.56-1.28)$ & 0.429 & $1.38(0.77-2.49)$ & 0.278 \\
AJCC stage & 1 & $4.06(0.98-16.90)$ & 0.054 & $7.92(1.59-39.34)$ & 0.011 \\
\hline
\end{tabular}

*Adjusted for age, sex, TSH, and BMI

OR Odds ratio, BMI Body mass index, AJCC American Joint Committee on Cancer, and MetS Metabolic syndrome dysmetabolic condition than of a specific dysmetabolic trait. More invasive characteristics including more lymph node metastasis and later AJCC stage were also observed in

$\begin{array}{lll}\text { Factors and subgroup } & \\ \text { Blood pressure } 130 / 85 \mathrm{mmHg} \\ \text { Tumor size } \\ \text { Lymph node metastasis } \\ \text { Multifocal tumor } \\ \text { AJCC stage } \\ \text { Diabetes } \\ \text { Tumor size } \\ \text { Lymph node metastasis }\end{array}$

patients with MetS. Blood pressure $\geq 130 / 85 \mathrm{mmHg}$ and low HDL were risk factors for larger tumor and lymph node metastasis. 
Previous studies had well defined the relationship between MetS and thyroid cancer. MetS and each component of the MetS are associated with an increased risk of thyroid cancer. Few studies focused on the effects of MetS on the aggressiveness and prognosis of thyroid cancer. A study by $\mathrm{Li}$ et al. demonstrated that multifocal tumor and extrathyroidal extension were more common in patients with diabetes. But there was no significant difference in tumor size and lymph node metastasis between patients with diabetes and the control group, which was similar with the results in our study [7]. Li et al. carried out a comparative analysis of the serum proteome profiles of thyroid cancer. In their study, TG and HDL levels were significantly different between patients with thyroid cancer and control groups. And among patients with PTC, a low HDL level was associated with lymph node metastasis. No significant association was observed between TG level and lymph node metastasis [8]. Epidemiology studies have reported the positive association between obesity and thyroid cancer risk. Correlation between BMI and the aggressiveness of PTC had been reported in several studies, but the results were inconsistent [9-11]. In the study by Kim et al., a higher BMI was associated with more aggressive tumor features, such as lymph node metastasis, lymphatic invasion, and tumor multiplicity in patients $\geq 45$ years of age [10]. However, Matrone et al. did not demonstrate any association between BMI and aggressiveness of thyroid cancer in 1058 patients [11]. In another study by Grani et al., they detected no association between obesity and the aggressive features of thyroid cancer [12]. BMI was used as a major measurement in most of the studies. But body fat distribution and an impaired adipose tissue function, rather than total fat mass, could better predict insulin resistance and related complications at the individual level [6]. Abdominal adiposity might play a more significant role in thyroid carcinogenesis and the progression of thyroid cancer. Few studies focused on the impact of abdominal adiposity on aggressiveness of PTC. In our study, we did not demonstrate a positive association between abdominal adiposity and aggressiveness of PTC. However, we are not aware of any previous studies of hypertension and aggressiveness of PTC. We found that blood pressure $\geq 130 / 85 \mathrm{mmHg}$ was associated with larger tumor, lymph node metastasis, and later AJCC stage.

Several biologic mechanisms may explain the link between MetS and the aggressiveness of PTC. First, insulin resistance is a key factor in the pathophysiology of MetS. It was reported that insulin assist many tumors' generation and progression. An increased level of insulin may stimulate vascular endothelial growth factor expression and promote the proliferation of vascular endothelial cells in cancer [13]. Insulin resistance can increase proliferation, angiogenesis, cellular mobility, apoptosis, and damage to DNA molecules of cancer cells by active oxygen forms due to excess glucose. Furthermore, thyroid cancer cells overexpress both insulin-like growth factor (IGF) ligands and their receptors. The IGF axis which was activated by insulin resistance in MetS can promote cell proliferation and inhibit programmed cell death [14]. Second, TSH is involved in mitogenic pathways of the thyroid gland. Cross-sectional studies have found correlations between TSH and the components of the MetS $[15,16]$. Previous studies indicated that the prevalence of dyslipidemia increased along with higher TSH concentrations [16, 17]. In our study, the TSH level was higher but not statistically significant in patients with MetS. Increased serum TSH levels may stimulate thyroid cell proliferation and growth, and promote the progression of thyroid cancer. Third, the low-grade chronic and systemic inflammation in MetS may also affect the aggressiveness of thyroid cancer. MetS could result in NF$\kappa \mathrm{B}$ activation and increase the synthesis and secretion of chemokines which played a central role in the progression of thyroid cancer [18-20]. Other factors, such as increased levels of estradiol, may also play important role in thyroid cancer progression [21].

In the present study, we investigated the association between MetS and aggressiveness of PTC and found that MetS was associated with aggressiveness of PTC. There were several limitations in our study. We included a relatively small number of patients in this study. Due to the limited duration of follow-up, our study has no information about follow-up evaluation of the patients for the potential development of future recurrence or metastases. In addition, some potential confounding factors (i.e., duration of MetS, duration and treatment, smoke) were not taken into account in our analysis.

In conclusion, in our hospital-based cohort study MetS was associated with the aggressiveness of PTC. This association was still significant after adjusted for age, sex, TSH, and BMI. MetS is a reversible condition associated with modern lifestyle. Dietary adjustment may help to regress MetS and the aggressiveness of PTC.

\section{Data availability}

The datasets generated during and/or analysed during the current study are available from the corresponding author on reasonable request.

Author contributions J.L.S. and L.R.L.: study design, analysis of data and drafting the work. X.Z.Y. and L.Z.: acquisition and analysis of data, Z.L.X., J.J.L., and S.R.S.: interpretation of data. C.C. supervised the study and approved the final manuscript.

\section{Compliance with ethical standards}

Conflict of interest The authors declare no competing interests. 
Ethical approval This study was performed with the approval of the ethics committee of the Renmin Hospital of Wuhan University.

Informed consent Informed consent was obtained from all individual participants included in the study.

Publisher's note Springer Nature remains neutral with regard to jurisdictional claims in published maps and institutional affiliations.

\section{References}

1. W.J. Wei, G.Q. Zhang, Q.Y. Luo, Postsurgical management of differentiated thyroid cancer in China. Trends Endocrinol. Metab. 29, $71(2018)$

2. C.D. Seib, J.A. Sosa, Evolving understanding of the epidemiology of thyroid cancer. Endocrinol. Metab. Clin. North Am. 48, 23 (2019)

3. M.G. Saklayen, The global epidemic of the metabolic syndrome. Curr. Hypertens. Rep. 20, 12 (2018)

4. D.T. Yin, H. He, K. Yu, J. Xie, M. Lei, R. Ma, H. Li, Y. Wang, Z. Liu, The association between thyroid cancer and insulin resistance, metabolic syndrome and its components: a systematic review and meta-analysis. Int. J. Surg. 57, 66 (2018)

5. J.H. Park, M. Choi, J.H. Kim, J. Kim, K. Han, B. Kim, D.H. Kim, Y.G. Park, Metabolic syndrome and the risk of thyroid cancer: a nationwide population-based cohort study. Thyroid. 30, 1496 (2020)

6. M.H. Kim, J.Y. Huh, D.J. Lim, M.I. Kang, Does the risk of metabolic syndrome increase in thyroid cancer survivors? Thyroid. 27, 936 (2017)

7. C. Li, J. Kuang, Y. Zhao, et al. Effect of type 2 diabetes and antihyperglycemic drug therapy on signs of tumor invasion in papillary thyroid cancer. Endocrine. 69, 92-99 (2020)

8. D. Li, L. Zhou, C. Ma, W. Chen, Y. Zhang, S. Yu, D. Wang, Y. Zou, J. Wu, L. Qiu, Comparative analysis of the serum proteome profiles of thyroid cancer: An initial focus on the lipid profile. Oncol. Lett. 18, 3349 (2019)

9. C. Li, L. Zhou, G. Dionigi, F. Li, Y. Zhao, H. Sun, The association between tumor tissue calcification, obesity, and thyroid cancer invasiveness in a cohort study. Endocr. Pract. 26, 830 (2020)

10. S.H. Kim, H.S. Park, K.H. Kim, H. Yoo, B.J. Chae, J.S. Bae, S.S. Jung, B.J. Song, Correlation between obesity and clinicopathological factors in patients with papillary thyroid cancer. Surg. Today 45, 723 (2015)

11. M. Antonio, C. Giovanni, B. Marianna, et al. Potential impact of BMI on the aggressiveness of presentation and clinical outcome of differentiated thyroid cancer. J. Clin. Endocrinol. Metab. 105, e1124-e1134 (2020)

12. G. Grani, L. Lamartina, T. Montesano et al. Lack of association between obesity and aggressiveness of differentiated thyroid cancer. J. Endocrinol. Invest. 42(1), 1-6 (2018)

13. C. Miele, J.J. Rochford, N. Filippa, S. Giorgetti-Peraldi, Van, E. Obberghen, Insulin and insulin-like growth factor-I induce vascular endothelial growth factor mRNA expression via different signaling pathways. J Biol. Chem. 275, 21695 (2000)

14. L. Manzella, M. Massimino, S. Stella, E. Tirro, M.S. Pennisi, F. Martorana, G. Motta, S.R. Vitale, A. Puma, C. Romano, S. Di Gregorio, M. Russo, P. Malandrino, P. Vigneri, Activation of the IGF axis in thyroid cancer: implications for tumorigenesis and treatment. Int. J. Mol. Sci. 20, 3258 (2019)

15. A. Roos, S.J. Bakker, T.P. Links, R.O. Gans, B.H. Wolffenbuttel, Thyroid function is associated with components of the metabolic syndrome in euthyroid subjects. J Clin Endocrinol Metab 92, 491 (2007)

16. J.J. Garduno-Garcia, U. Alvirde-Garcia, G. Lopez-Carrasco, M.M. Padilla, R. Mehta, O. Arellano-Campos, R. Choza, L. Sauque, M. E. Garay-Sevilla, J.M. Malacara, F.J. Gomez-Perez, C.A. AguilarSalinas, TSH and free thyroxine concentrations are associated with differing metabolic markers in euthyroid subjects. Eur. J. Endocrinol. 163, 273 (2010)

17. A. Shinkov, A.M. Borissova, R. Kovatcheva, I. Atanassova, J. Vlahov, L. Dakovska, The prevalence of the metabolic syndrome increases through the quartiles of thyroid stimulating hormone in a population-based sample of euthyroid subjects. Arq. Bras. Endocrinol. Metabol. 58, 926 (2014)

18. G. Revilla, L. Cedo, M. Tondo, A. Moral, J.I. Perez, R. Corcoy, E. Lerma, V. Fuste, S.T. Reddy, F. Blanco-Vaca, E. Mato, J.C.L.D. L. Escola- Gil, HDL and endocrine-related cancer: from pathogenic mechanisms to therapies. Semin. Cancer Biol. 2020.

19. A.R. Saltiel, J.M. Olefsky, Inflammatory mechanisms linking obesity and metabolic disease. J. Clin. Invest. 127, 1 (2017)

20. M. Tafani, E. De Santis, L. Coppola, G.A. Perrone, I. Carnevale, A. Russo, B. Pucci, A. Carpi, M. Bizzarri, M.A. Russo, Bridging hypoxia, inflammation and estrogen receptors in thyroid cancer progression. Biomed. Pharmacother. 68, 1 (2014)

21. M. Derwahl, D. Nicula, Estrogen and its role in thyroid cancer. Endocr. Relat. Cancer 21, T273 (2014) 